\title{
INTRODUCTION
}

\section{Executive functions and academic learning}

\author{
Patrice Potvin ${ }^{1 *} \&$ Grégoire Borst ${ }^{2}$
}

\footnotetext{
${ }^{1}$ Université du Québec à Montréal, Faculty of Education Sciences, Department of Didactics, Montreal, Canada

${ }^{2}$ Université Paris Descartes, LaPsyDé, Paris, France

*Author email address: potvin.patrice@uqam.ca
}

To cite this article: Potvin, P., \& Borst, G. (2018). Executive functions and academic learning. Neuroeducation, 5(2), 42-43.

DOI: https://doi.org/10.24046/neuroed.20180502.42

All rights reserved (C) 2018 - Association for Research in Neuroeducation / Association pour la recherche en neuroéducation 
It is increasingly recognized that executive functions (inhibitory control, attention, updating, mental flexibility, reasoning, planning) play an important role in the educational paths of pupils and students. Indeed, learners who present the most developed executive functions are often those who perform and succeed best in school and university. They are also those with the least problems of behavior. However, some studies have also been able to establish convincing links between certain executive functions, which are general in scope, and some more specific difficulties identified by experts in didactics or in special education. Several important questions emerge: How are the EFs involved in such or such disciplinary or specific performance? How can teachers, and more broadly the school systems, take knowledge of EFs into account in their efforts to support learners? Are EFs mere causes of academic learning or can they, in turn, be the effects? Etc.

In this special issue, the Neuroeducation journal has invited researchers to present results of their studies and arguments that are based directly or indirectly on brain functions, and which will provide answers to one or more of these questions.

For example, Marie-Pier Godin, Andréanne Gagné and Nathalie Chapleau, in an article entitled "Phonographic spelling errors in developmental language disorder: Insights from executive functions", present the results of a study conducted on 32 children, some of whom suffered of a language disorder (DLD). The results suggest that some children with DLD have limitations in "updating in working memory", which may lead to slower spelling and more phonographic errors.

For their part, Stella Vosniadou, Dimitrios Pnevmatikos and Nikos Makris, in "The role of executive function in the construction and employment of scientific and mathematical concepts that require conceptual change learning", discuss the general importance of EFs in learning science and mathematics, and particularly in conceptual change processes. Based on their previous research, they illustrate, among other things, the role of inhibition in such processes.

In the results that Bénédicte Willame and Philippe Snauwaert present in their article entitled "Inhibitory control training and chemistry learning in upper secondary education: Fostering a change in conceptual prevalence" (free translation), convincing experimental evidence suggests that training courses designed to generate cognitive conflicts and train the learners in inhibitory control can have positive effects on the learning of chemistry concepts.

In an analysis of "Theoretical perspectives on executive functions in educational context in preschool children" (free translation), Stéphanie Duval, Noémie Montminy and Aimée Gaudette-Leblanc document the evolution of the theoretical considerations that nourish our understanding of the development of executive functions for children aged between 3 and 6 and come up with options for supporting EF skills at the preschool level.
Reuven Babai, Eldad Cohen and Ruth Stavy then present the results of an experimental study of proportional reasoning that compares the measurements obtained with two similar visual tasks that differ in that only one reduces the interference due to comparison of the numbers making up proportionality ratios. The article, titled "Proportional reasoning: Reducing the interference of natural numbers through an intervention based on the problem-solving framework of executive functions", suggests that modes of representation or the order of their presentation may have implications on the learning of proportionality.

And finally, François Thibault and Patrice Potvin, in "Executive function as a predictor of physics-related conceptual change", present the results of an experimental research of which data support the hypothesis that the engineering students who present the best EFs are also those who, more often, will undergo conceptual changes during university courses in mechanics.

In the end, this special issue brings an unprecedented and convincing light on the links initially presumed between executive functions and academic learning. It illustrates how particular EFs seem to intervene in the resolution of distinct problems that come from disciplines that are often very different. It also allows catching a glimpse of the constraints and the hopes that could raise a possible education of executive functions. 